\title{
Tissue-specific function of Patj in regulating the Crumbs complex and epithelial polarity
}

\author{
Clothilde Pénalva and Vincent Mirouse*
}

\begin{abstract}
SUMMARY
Patj is described as a core component of the Crumbs complex. Along with the other components, Crumbs and Stardust, Patj has been proposed as essential for epithelial polarity. However, no proper in vivo genetic analysis of Patj function has been performed in any organism. We have generated the first null mutants for Drosophila Patj. These mutants are lethal. However, Patj is not required in all epithelia where the Crumbs complex is essential. Patj is dispensable for ectoderm polarity and embryonic development, whereas more severe defects are observed in the adult follicular epithelium, including mislocalisation of the Crumbs complex from the apical domain, as well as morphogenetic defects. These defects are similar to those observed with crumbs and stardust mutants, although weaker and less frequent. Also, gain-of-function of Crumbs and Patj mutation genetically suppress each other in follicular cells. We also show that the first PDZ domain of Patj associated with the Stardust-binding domain are sufficient to fully rescue both Drosophila viability and Crumbs localisation. We propose that the only crucial function of Patj hinges on the ability of its first two domains to positively regulate the Crumbs complex, defining a new developmental level of regulation of its dynamics.
\end{abstract}

KEY WORDS: Crumbs, Epithelium, Follicular cells, Polarity

\section{INTRODUCTION}

Epithelial polarity relies on the dynamic interplay of several protein complexes that define the different cortical domains (Laprise and Tepass, 2011; St Johnston and Ahringer, 2010). Among these, the Crumbs (Crb) complex plays key roles in the definition of the apical domain and in the organisation and positioning of the first apical junction, which are adherens junctions in Drosophila and tight junctions in mammals (Tepass et al., 1990; Wodarz et al., 1995; Bulgakova and Knust, 2009; Morais-de-Sá et al., 2010; Bazellieres et al., 2009; Hurd et al., 2003). At the molecular level, essential components of the Crb complex are Crb, Stardust (Sdt; PALS1) and Patj. Crb is a transmembrane protein; its cell polarity function depends on a C-terminal PDZ binding motif (ERLI) that binds Sdt (Klebes and Knust, 2000; Bachmann et al., 2001; Hong et al., 2001; Roh et al., 2002). Patj has been isolated as a component of the Crb complex via direct interaction with Sdt, involving heterodimerisation of their L27 domains (Roh et al., 2002; Bulgakova et al., 2008). To mediate its function in polarity, the Crb complex also interacts in multiple ways with other polarity determinants, including the PAR6/aPKC complex (Hurd et al., 2003; Kempkens et al., 2006; Lemmers et al., 2004; Krahn et al., 2010). These interactions together reflect the intricate dynamics of these complexes, which have yet to be fully elucidated.

There are genetic data clearly showing the importance of $\mathrm{Crb}$ and Sdt, but less is known about Patj. Reducing PATJ protein levels in mammal cell culture leads to destabilisation of the Crb complex and to defects in tight junction assembly (Lemmers et al., 2002; Michel et al., 2005; Shin et al., 2005). However, this phenotype is mild compared with those of $C r b$ or $S d t$ mutants, and one could

GReD Laboratory, UMR CNRS 6293, Clermont Université, INSERM U1103, Faculté de Médecine, place Henri-Dunant, 63000 Clermont-Ferrand, France.

*Author for correspondence (vincent.mirouse@u-clermont1.fr)

Accepted 21 September 2012 assume that the difference reflects hypomorphic conditions. Genetic data in fruit flies have failed to produce any clear picture of Patj function, although the gene appears to be essential for viability (Bhat et al., 1999; Nam and Choi, 2006; Pielage et al., 2003; Richard et al., 2006).

Here we present the first null mutants for the Drosophila Patj gene. This analysis reveals a tissue-specific requirement for the stability of the Crb complex at the apical membrane.

\section{MATERIALS AND METHODS}

\section{Drosophila genetics}

Patj mutants were generated by imprecise excision of $P\{G S V 2\} G S 50262$. Excisions that were lethal when crossed with the $D f(3 L) M y 10$ deficiency were analysed at the molecular level. Only deletions exclusively affecting Patj were retained $\left(\right.$ Patj $^{53}$, Patj $^{58}$, Patj $\left.^{63}\right)$ and recombined with FRT2A. Rescue by a Patj transgene was assessed for all alleles.

Clonal analyses were performed using the flp/FRT or MARCM systems with GFP as marker for wild-type or mutant cells, respectively. Flies were dissected 4 days after clone induction by heat shock. Mutant stocks are FRT2A, Patj, FRT19A, sdt ${ }^{K 85}$ (Berger et al., 2007) and FRT82B, $c r b^{l}$ (Tepass et al., 1990). Overexpression was performed using Tub:Gal4 flipout or MARCM systems. UAS transgenes are UASt:Patj (Bhat et al., 1999) and UASt:crb ${ }^{W T-30.12 e}$ (Wodarz et al., 1995). For embryo analysis, maternal contribution was removed using the flp/FRT Ovo ${ }^{\mathrm{D} 1}$ system and zygotic mutants were selected by the absence of TM3, twi:Gal4, UAS:eGFP.

\section{Transgenes}

The Patj genomic rescue transgene was produced by cloning a genomic PCR fragment into the pCASPER4 vector. Patj transgenes for structure/function analysis, including a full-length transgene, were produced by PCR on Patj cDNA and cloning with and without GFP for Cterminal fusions in pWRpAUbiqP, which contains a Ubiquitin-63E promoter. Transgenic flies were generated by Fly Facility.

\section{Immunostaining}

Immunofluorescent staining was performed according to standard protocols using the following primary antibodies: rabbit anti-PKCz $(1 / 500 ; \mathrm{C}-20$, Santa Cruz), mouse anti-Dlg (1/50; Developmental Studies Hybridoma Bank, University of Iowa), rabbit anti-Baz (1/1000) (Wodarz et al., 1999), 
rat anti-Crb (1/500) (Pellikka et al., 2002), and rabbit anti-Patj (1/500) (Tanentzapf et al., 2000). Images were acquired on a Leica SP5 microscope.

\section{RESULTS AND DISCUSSION}

\section{Patj null mutations are lethal}

No proper mutation of the Patj gene was available, limiting its genetic manipulation. In order to generate Patj mutants, 100 excisions of a $P$-element-based transgene inserted in the $5^{\prime}$ region of Patj were produced (Fig. 1A). We identified three internal deletions of Patj associated with lethality. Patj ${ }^{58}$ and Patj $^{63}$ delete start codons, whereas $\mathrm{Patj}^{53}$ shows complete deletion of the coding sequence. A rescue construct containing the Patj gene and $300 \mathrm{bp}$ upstream of its transcription start site perfectly rescued the lethality and fertility of Patj mutants. We therefore assumed these alleles to be null mutations for Patj, and we confirmed the absence of Patj protein in mutant follicle cells as compared with the surrounding wild-type cells (Fig. 1B). Our results confirm previous findings that Patj is an essential gene (Nam and Choi, 2006; Richard et al., 2006). However, despite previous reports that Patj mutants die during the second instar larval stage, we found that Patj homozygotes or transheterozygotes could reach the pupal stage, highlighting the utility of bona fide null mutants. We also produced maternal Patj germline clones to test whether maternal contribution could mask embryonic phenotype. However, maternal and zygotic null mutants were able to reach the pupal stage, like the zygotic mutants, indicating that Patj is dispensable for embryonic development. In addition, we failed to detect polarity defects in the embryonic ectoderm upon examining the localisation of $\mathrm{Crb}$ and other polarity markers (Fig. 1C,D; data not shown).
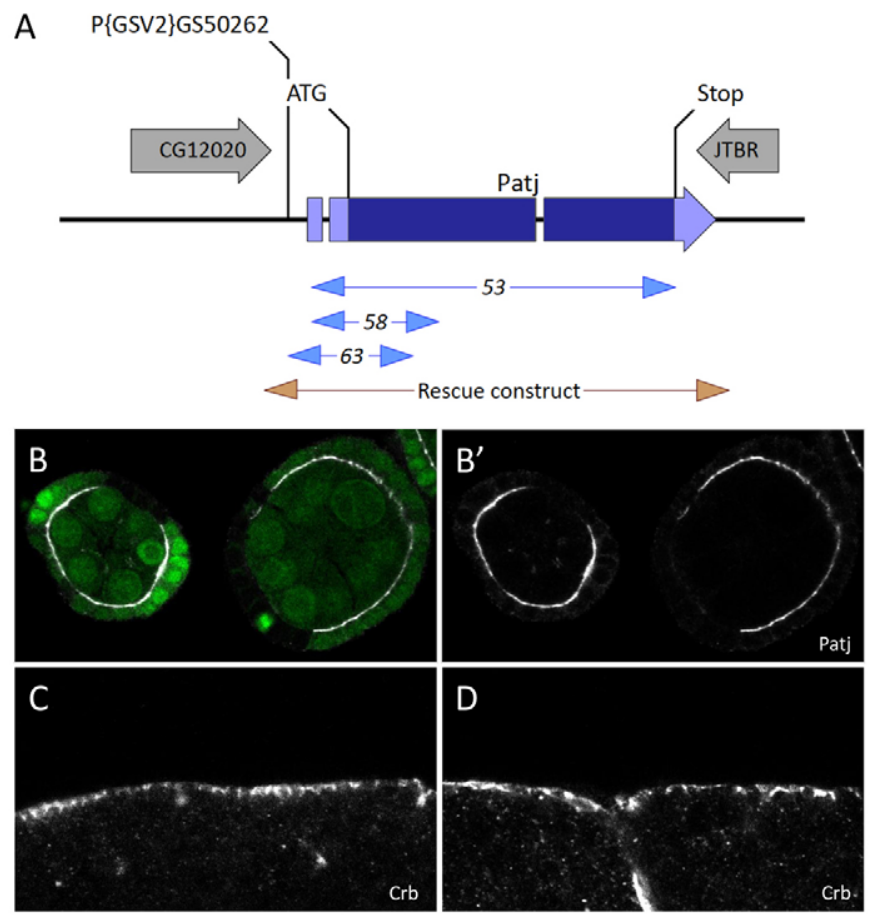

Fig. 1. Null mutants for Patj. (A) The Drosophila Patj locus. The site of the $P\{G S V 2\} G S 50262$ insertion, deletion mutations and the rescue transgene are indicated. (B, $\mathbf{B}^{\prime}$ ) Mutant clones for Patj ${ }^{53}$ in follicular cells stained for Patj (white). Mutant cells are marked by the absence of GFP (green). (C,D) Normal apical localisation of Crb in zygotic (C) or maternal zygotic (D) embryos mutant for Patj.

\section{Patj regulates Crb complex stability}

We therefore analysed Patj function in another tissue; we looked at the follicular epithelium that surrounds the germline cysts of the ovary. We first investigated the localisation of the other Crb complex members $\mathrm{Crb}$ and Sdt. Both showed partial mislocalisation from the apical domain in Patj mutant as compared with wild-type cells. This phenotype is usually weak during early stages (1-5), but tends to gain strength later, leading in some cases to a complete apical loss of both Sdt and Crb, thus indicating that Patj is required for Crb complex stability at the apical domain (Fig. 2A,B). Absence of Crb and Sdt from the apical domain is not associated with a strong cytoplasmic accumulation of these proteins, suggesting that they might be degraded. Because Crb and Sdt are important for defining the apical domain of epithelial cells, we also looked at the localisation of other polarity markers. aPKC is also less apically enriched in Patj mutant cells than in wild-type cells, although the effect on this key polarity determinant is weaker than that observed for Sdt and Crb (Fig. 2C,D). Baz protein is also globally absent from the apical domain, but remains in subapical spots that might correspond to adherens junctions (Fig. 2E) (Morais-de-Sá et al., 2010). Also, we did not observe any lateral markers, such as Discs large (Dlg), extending apically in Patj mutant cells, which is usually the case when the apical domain is no longer specified (Fig. 2F).

In agreement with these results, we found extremely rare cases $(<1 \%)$ in which a loss of polarity of Patj mutant cells was indicated by the formation of multilayers or round cells (Fig. 2H). However, we observed a cell flattening in Patj mutant clones compared with wild-type cells in $\sim 20 \%$ of the follicle-containing clones (Fig. 2F,G). This flattening always affected cells on the lateral side of the follicles. We compared the Patj phenotype with those of $\mathrm{crb}$ and $s d t$ mutants (Fig. 2I; supplementary material Figs S1, S2). As previously described, $c r b$ mutation frequently induced multilayers $(42 \%)$ and cell flattening (22\%) (Tanentzapf et al., 2000). $s d t$ mutation also induced the same defects, with multilayers (31\%) and some flat cells in the epithelium (37\%). Moreover, $c r b$ and $s d t$ mutant cells showed a greater loss of apical markers than Patj cells (supplementary material Figs S1, S2). Taken together, our data suggest that the flattening is a mild phenotype, whereas multilayer formation corresponds to a stronger polarity phenotype. Our results therefore suggest that the loss of any one of the three core components of the Crb complex leads to similar phenotypic traits, but with the severity of defects decreasing in the order $c r b, s d t$, Patj. The importance of $\mathrm{Crb}$ as a polarity determinant is also apparent through the extension of the apical domain and disruption of epithelial architecture when $\mathrm{Crb}$ is overexpressed (Tanentzapf et al., 2000; Wodarz et al., 1995). Mirroring the loss-of-function experiments, Sdt overexpression in follicle cells leads to less pronounced defects than Crb overexpression (Horne-Badovinac and Bilder, 2008). Similar clonal overexpression of Patj did not induce any polarity defect in follicle cells (Fig. 2J; data not shown), confirming that Patj plays a more subtle role in epithelial polarity than Crb and Sdt.

\section{The L27 and first PDZ domain are sufficient for Patj function}

It has been shown that the L27 domain and the first of the four PDZ domains of Patj are able to rescue lethality associated with Patj deletion (Nam and Choi, 2006). We produced several Patj transgenes to provide a more accurate structure-function analysis. A full-length Patj transgene compared with a transgene encoding these two domains exhibited no differences; both were able to fully 

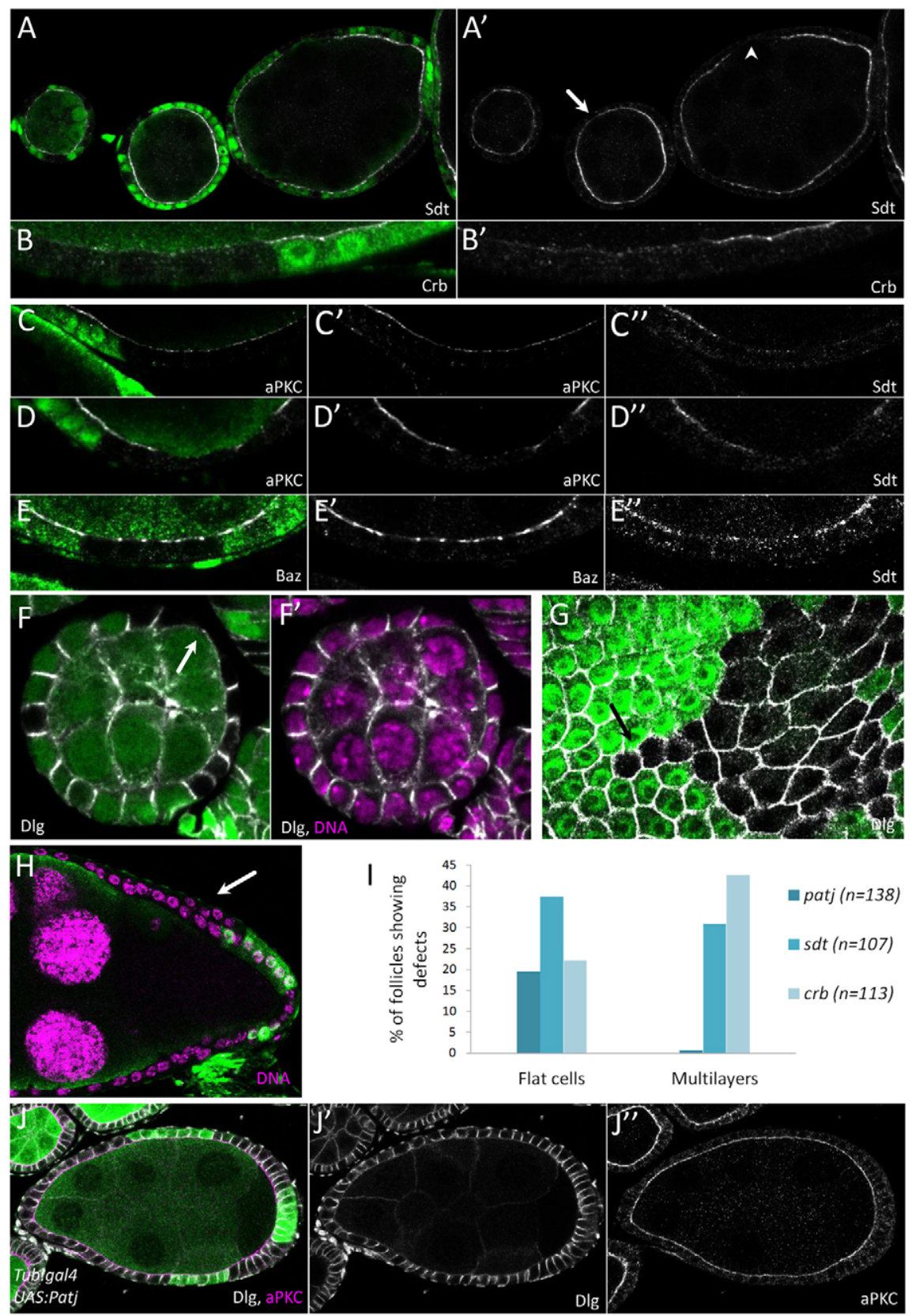

Fig. 2. Patj mutant phenotype in the

follicular epithelium. (A-H) Patj null mutation clones viewed in transverse $(A-F, H)$ or planar $(G)$ section stained (white) for Sdt $\left(A, A^{\prime}, C^{\prime \prime}, D^{\prime \prime}, E^{\prime \prime}\right)$, $\mathrm{Crb}\left(\mathrm{B}, \mathrm{B}^{\prime}\right)$, aPKC $\left(\mathrm{C}, \mathrm{C}^{\prime}, \mathrm{D}, \mathrm{D}^{\prime}\right), \mathrm{Baz}\left(\mathrm{E}, \mathrm{E}^{\prime}\right)$, Dlg (F$G$ ) and for DNA (purple, $F^{\prime}, H$ ). Mutant cells are marked by the absence of GFP (green). ( $A, A^{\prime}$ ) Sdt loss from the apical domain can be partial (arrows) or complete (arrowhead). (B-E") Reduction in the apical domain of Crb, aPKC and $\mathrm{Baz}$ is also observed. (F-G) Patj mutant clones showing a cell flattening defect. This defect is not observed in all the cells, which is in part owing to the genotype of the surrounding cells (arrow in G). (H) Patj mutant clone forming a multilayer (arrow). (I) Quantification of Patj, sdt and crb phenotypes in follicular cells. Percentages of follicles containing mutant clones and showing multilayers or cell flattening. (J-J") Clonal overexpression of Patj (green cells) does not induce any polarity defect in follicular cells as visualised with Dlg $\left(\mathrm{J}^{\prime}\right.$, white in $\mathrm{J})$ and aPKC (J", purple in J). rescue Patj mutant-associated lethality (Fig. 3A,B). In addition, we investigated $\mathrm{Crb}$ localisation in follicle cells mutant for Patj in flies carrying these transgenes. The presence of full-length Patj or L27PDZ1 proteins fully rescued the Crb complex localisation and cell flattening phenotype (Fig. 3A,B). By contrast, transgenes encoding only L27 or only PDZ1 failed to rescue lethality, Crb localisation or cell flattening in follicle cells mutant for endogenous Patj (Fig. 3C,D). A similar inability to rescue the lethality was observed in flies expressing L27 and PDZ1 as encoded by two distinct transgenes (Fig. 3E). Taken together, these results show that all the essential functions of Patj are concentrated in these two domains and that they have to be linked together. Also, the ability of Patj transgenes to rescue lethality is correlated with their ability to restore apical localisation of the Crb complex, suggesting that this constitutes the main function of Patj.

Analysis of the subcellular localisation of the different Patj domains fused to GFP indicates that the PDZ1 domain localises on its own to the apical domain, although less efficiently than proteins containing the L27 domain, suggesting that PDZ1 can bind an apical protein (Fig. 3A-D). Whereas L27 domain binding to Sdt is well established, what binds to the PDZ1 domain is far less clear. No partner has been identified in flies, and only a few proteins are proposed to link to the mammalian PATJ PDZ2 domain, which corresponds to fly PDZ1. One of these is TSC2 (Massey-Harroche et al., 2007), but we failed to find similar interaction between the Drosophila proteins. Mammalian PATJ PDZ2 also interacts with angiomotin (AMOT), and this interaction seems robust because it is conserved with the other members of the AMOT protein family and the mammalian paralogue of PATJ called MUPP1 (MPDZ) (Sugihara-Mizuno et al., 2007; Wells et al., 2006). However, the Drosophila genome does not contain any member of the AMOT family. These data suggest the existence of an as yet unidentified apical ligand of PDZ1 that is essential for Patj function. 


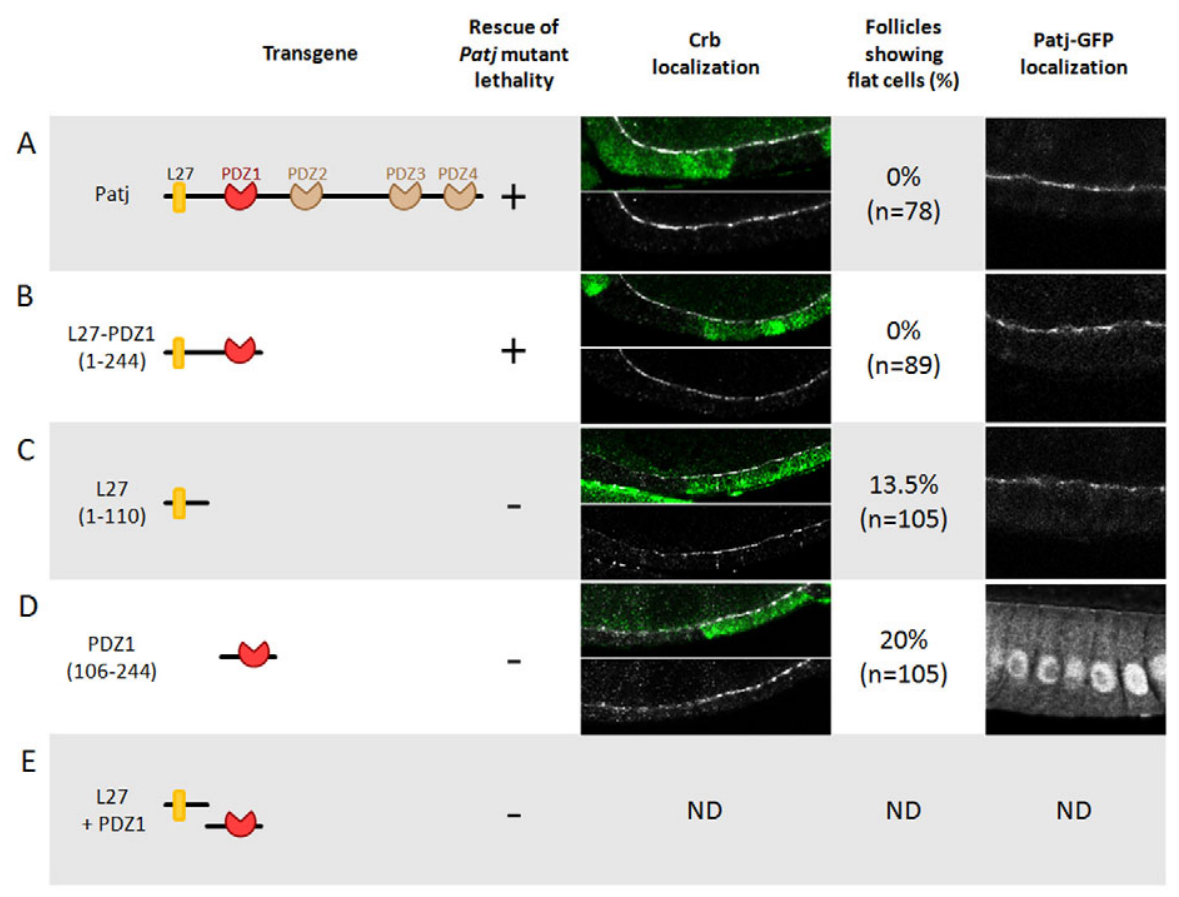

Fig. 3. Structure-function analysis of Patj. (A) Full-length Patj, (B) L27 + PDZ1 domains, (C) L27 domain, (D) PDZ1 domain, (E) L27 and PDZ1 domains encoded by two independent transgenes. The ability of these transgenes to rescue Patj mutant lethality is indicated. Patj mutant clones in the follicular epithelium are marked by the absence of GFP and stained for Crb (white) in flies containing the different transgenes. Quantification of the cell flattening phenotype is shown. The L27 and PDZ1 domains are necessary and sufficient for rescue of $\mathrm{Crb}$ localisation and lethality. The final column illustrates the subcellular localisation in the follicular cells of the different Patj proteins as visualised by GFP. ND, not determined.

\section{Genetic interaction between Patj and Crb}

All the defects caused by Patj mutation are therefore also present in other mutants of the Crb complex, and at least one of the two key domains of Patj interacts directly with Sdt. Taken together, these results suggest that the main function of Patj is to regulate the Crb complex. We took advantage of the moderate effect of Patj mutation on follicle cell polarity to look for genetic interaction with a $\mathrm{Crb}$ gain-of-function. We analysed the phenotype of Patj
MARCM mutant clones overexpressing $\mathrm{Crb}$, and compared their effects with both Patj mutant and Crb overexpression on their own. As previously described, Crb overexpression disrupts follicular cell polarity, as seen by extension of aPKC localisation to the lateral domain and from the spheroidal shape of the cells, and can lead to multilayer formation (Fig. 4A; data not shown) (Tanentzapf et al., 2000). Crb overexpression also blocks the cuboid-to-squamous transition of a subpopulation of follicular cells, termed stretched
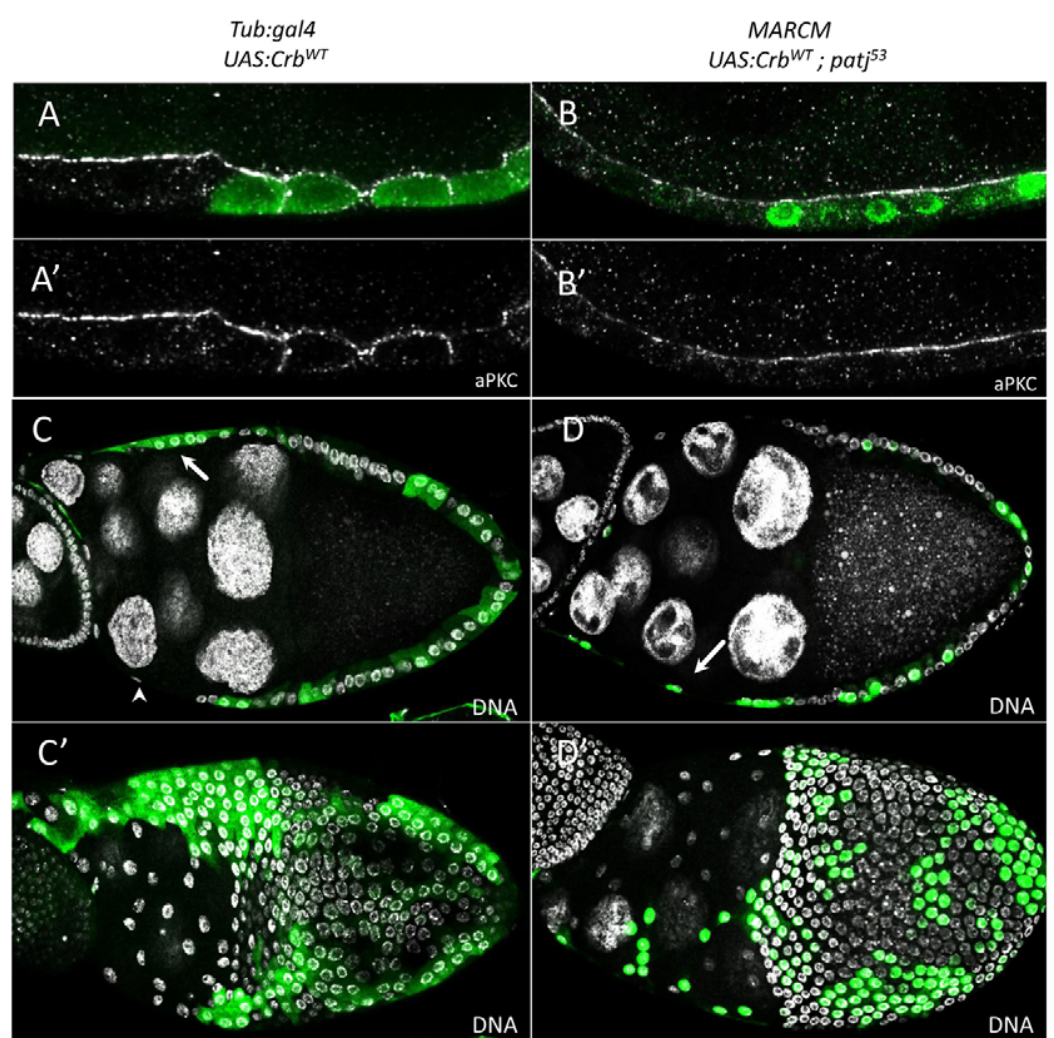

DNA
Fig. 4. Genetic interaction between Patj and crb. Clonal overexpression (green, positive cells) of $\mathrm{Crb}$ in wild-type $(A, C)$ or Patj mutant $(B, D)$ cells. $\left(\mathbf{A}, \mathbf{A}^{\prime}\right) \mathrm{Crb}$ overexpression induces a loss of cell polarity that can be visualised by an extension of aPKC cortical staining and by the spheroidal shape of the cells. (B, $\left.\mathbf{B}^{\prime}\right)$ This phenotype is suppressed when the cells are mutant for Patj. ( $\left.\mathbf{C}, \mathbf{C}^{\prime}\right)$ Transverse section (C) and top view $\left(\mathbf{C}^{\prime}\right)$. $\mathbf{C r b}$ overexpression blocks cell flattening of anterior cells at stage 9 of oogenesis (arrow compared with arrowhead). $\left(\mathbf{D}, \mathbf{D}^{\prime}\right)$ Transverse section (D) and top view ( $\left.\mathrm{D}^{\prime}\right)$. This defect is suppressed when the cells are mutant for Patj. Also, Crb overexpression rescues the various Patj mutant defects observed in follicular cells (see Fig. 2). 
cells, at stage 9 of oogenesis (Fig. 4C) (Grammont, 2007). Patj MARCM mutant clones overexpressing $\mathrm{Crb}$ exhibit normal morphology, and aPKC no longer extends to the lateral domain of the cells, even though it is slightly more enriched on the apical domain (Fig. 4B). Thus, we never observed a reduction in aPKC at the apical domain, in contrast to Patj mutant cells. In addition, $\mathrm{Crb}$ overexpression also fully rescues the cell flattening that results from the loss of Patj, whereas the absence of Patj restores the normal stretching of anterior cells at stage 9 (Fig. 4D). Thus, Crb overexpression and null mutation for Patj suppress each other for all the defects that we observed in follicle cells. These genetic data lead to the conclusion that the essential function of Patj is to positively regulate the Crb complex.

The Patj mutant phenotype suggests that it might be important for delivering or stabilising $\mathrm{Crb}$ at the apical membrane or for promoting its recycling from endosomes. PATJ knockdown in human cells leads to CRB mislocalisation from the apical cortex and its accumulation in early endosomes (Michel et al., 2005). Interestingly, Rab11 and the retromer are both important for $\mathrm{Crb}$ recycling in Drosophila (Pocha et al., 2011; Roeth et al., 2009; Zhou et al., 2011). However, they exercise this function in the embryo, where Patj is largely dispensable, indicating that Patj is not essential for Crb recycling.

Patj requirement appears stronger in some epithelia than in others, with no impact on embryonic ectoderm polarity but stronger defects in the follicular epithelium. Structure-function analyses of Sdt and Crb lead to similar conclusions, with the requirements for Sdt or Crb domains seeming to differ from one epithelial tissue to another (Wodarz et al., 1995; Bit-Avragim et al., 2008; Bulgakova and Knust, 2009; Fletcher et al., 2012). The reason for such differences remains to be elucidated. The Crb complex is also involved in the control of cell proliferation and cell morphogenetic processes other than the establishment or maintenance of epithelial polarity (Bulgakova and Knust, 2009; Grusche et al., 2010; Kerman et al., 2008; Laprise et al., 2010; Letizia et al., 2011; Xu et al., 2008). Modulation of its activity by Patj might participate in these developmental functions of the Crb complex.

\section{Note added in proof}

During the editorial process, another article was published describing Drosophila Patj mutants and their phenotypic analysis (Zhou and Hong, 2012).

\section{Acknowledgements}

We thank E. Knust, U. Tepass, A. Wodarz, K. W. Choi and S.-C. Nam for providing flies and reagents. We also thank the ICCF Confocal Microscopy Facility at Clermont University.

\section{Funding}

C.P. and V.M. are supported by an ATIP-Avenir grant, the Association pour la Recherche sur le Cancer (ARC) and the Fondation pour la Recherche Médicale (FRM).

\section{Competing interests statement}

The authors declare no competing financial interests.

\section{Supplementary material}

Supplementary material available online at

http://dev.biologists.org/lookup/suppl/doi:10.1242/dev.085449/-/DC1

\section{References}

Bachmann, A., Schneider, M., Theilenberg, E., Grawe, F. and Knust, E.

(2001). Drosophila Stardust is a partner of Crumbs in the control of epithelial cell polarity. Nature 414, 638-643.

Bazellieres, E., Assemat, E., Arsanto, J. P., Le Bivic, A. and Massey-Harroche,

D. (2009). Crumbs proteins in epithelial morphogenesis. Front. Biosci. 14, 21492169.
Berger, S., Bulgakova, N. A., Grawe, F., Johnson, K. and Knust, E. (2007). Unraveling the genetic complexity of Drosophila stardust during photoreceptor morphogenesis and prevention of light-induced degeneration. Genetics 176, 2189-2200.

Bhat, M. A., Izaddoost, S., Lu, Y., Cho, K. O., Choi, K. W. and Bellen, H. J. (1999). Discs Lost, a novel multi-PDZ domain protein, establishes and maintains epithelial polarity. Cell 96, 833-845.

Bit-Avragim, N., Hellwig, N., Rudolph, F., Munson, C., Stainier, D. Y. and Abdelilah-Seyfried, S. (2008). Divergent polarization mechanisms during vertebrate epithelial development mediated by the Crumbs complex protein Nagie oko. J. Cell Sci. 121, 2503-2510.

Bulgakova, N. A. and Knust, E. (2009). The Crumbs complex: from epithelial-cell polarity to retinal degeneration. J. Cell Sci. 122, 2587-2596.

Bulgakova, N. A., Kempkens, O. and Knust, E. (2008). Multiple domains of Stardust differentially mediate localisation of the Crumbs-Stardust complex during photoreceptor development in Drosophila. J. Cell Sci. 121, 2018-2026.

Fletcher, G. C., Lucas, E. P., Brain, R., Tournier, A. and Thompson, B. J. (2012). Positive feedback and mutual antagonism combine to polarize crumbs in the Drosophila follicle cell epithelium. Curr. Biol. 22, 1116-1122.

Grammont, M. (2007). Adherens junction remodeling by the Notch pathway in Drosophila melanogaster oogenesis. J. Cell Biol. 177, 139-150.

Grusche, F. A., Richardson, H. E. and Harvey, K. F. (2010). Upstream regulation of the hippo size control pathway. Curr. Biol. 20, R574-R582.

Hong, Y., Stronach, B., Perrimon, N., Jan, L. Y. and Jan, Y. N. (2001). Drosophila Stardust interacts with Crumbs to control polarity of epithelia but not neuroblasts. Nature 414, 634-638.

Horne-Badovinac, S. and Bilder, D. (2008). Dynein regulates epithelial polarity and the apical localization of stardust A mRNA. PLoS Genet. 4, e8.

Hurd, T. W., Gao, L., Roh, M. H., Macara, I. G. and Margolis, B. (2003). Direct interaction of two polarity complexes implicated in epithelial tight junction assembly. Nat. Cell Biol. 5, 137-142.

Kempkens, O., Médina, E., Fernandez-Ballester, G., Ozüyaman, S., Le Bivic, A., Serrano, L. and Knust, E. (2006). Computer modelling in combination with in vitro studies reveals similar binding affinities of Drosophila Crumbs for the PDZ domains of Stardust and DmPar-6. Eur. J. Cell Biol. 85, 753-767.

Kerman, B. E., Cheshire, A. M., Myat, M. M. and Andrew, D. J. (2008). Ribbon modulates apical membrane during tube elongation through Crumbs and Moesin. Dev. Biol. 320, 278-288.

Klebes, A. and Knust, E. (2000). A conserved motif in Crumbs is required for Ecadherin localisation and zonula adherens formation in Drosophila. Curr. Biol. 10, 76-85.

Krahn, M. P., Bückers, J., Kastrup, L. and Wodarz, A. (2010). Formation of a Bazooka-Stardust complex is essential for plasma membrane polarity in epithelia. J. Cell Biol. 190, 751-760.

Laprise, P. and Tepass, U. (2011). Novel insights into epithelial polarity proteins in Drosophila. Trends Cell Biol. 21, 401-408.

Laprise, P., Paul, S. M., Boulanger, J., Robbins, R. M., Beitel, G. J. and Tepass, U. (2010). Epithelial polarity proteins regulate Drosophila tracheal tube size in parallel to the luminal matrix pathway. Curr. Biol. 20, 55-61.

Lemmers, C., Médina, E., Delgrossi, M. H., Michel, D., Arsanto, J. P. and Le Bivic, A. (2002). hINADI/PATJ, a homolog of discs lost, interacts with crumbs and localizes to tight junctions in human epithelial cells. J. Biol. Chem. 277, 5408-5415.

Lemmers, C., Michel, D., Lane-Guermonprez, L., Delgrossi, M. H., Médina, E., Arsanto, J. P. and Le Bivic, A. (2004). CRB3 binds directly to Par6 and regulates the morphogenesis of the tight junctions in mammalian epithelial cells. Mol. Biol. Cell 15, 1324-1333.

Letizia, A., Sotillos, S., Campuzano, S. and Llimargas, M. (2011). Regulated $\mathrm{Crb}$ accumulation controls apical constriction and invagination in Drosophila tracheal cells. J. Cell Sci. 124, 240-251.

Massey-Harroche, D., Delgrossi, M. H., Lane-Guermonprez, L., Arsanto, J. P., Borg, J. P., Billaud, M. and Le Bivic, A. (2007). Evidence for a molecular link between the tuberous sclerosis complex and the Crumbs complex. Hum. Mol. Genet. 16, 529-536.

Michel, D., Arsanto, J. P., Massey-Harroche, D., Béclin, C., Wijnholds, J. and Le Bivic, A. (2005). PATJ connects and stabilizes apical and lateral components of tight junctions in human intestinal cells. J. Cell Sci. 118, 4049-4057.

Morais-de-Sá, E., Mirouse, V. and St Johnston, D. (2010). aPKC phosphorylation of Bazooka defines the apical/lateral border in Drosophila epithelial cells. Cell 141, 509-523.

Nam, S. C. and Choi, K. W. (2006). Domain-specific early and late function of Dpatj in Drosophila photoreceptor cells. Dev. Dyn. 235, 1501-1507.

Pellikka, M., Tanentzapf, G., Pinto, M., Smith, C., McGlade, C. J., Ready, D. F. and Tepass, U. (2002). Crumbs, the Drosophila homologue of human CRB1/RP12, is essential for photoreceptor morphogenesis. Nature 416, 143-149.

Pielage, J., Stork, T., Bunse, I. and Klämbt, C. (2003). The Drosophila cell survival gene discs lost encodes a cytoplasmic Codanin-1-like protein, not a homolog of tight junction PDZ protein Patj. Dev. Cell 5, 841-851. 
Pocha, S. M., Wassmer, T., Niehage, C., Hoflack, B. and Knust, E. (2011). Retromer controls epithelial cell polarity by trafficking the apical determinant Crumbs. Curr. Biol. 21, 1111-1117.

Richard, M., Grawe, F. and Knust, E. (2006). DPATJ plays a role in retinal morphogenesis and protects against light-dependent degeneration of photoreceptor cells in the Drosophila eye. Dev. Dyn. 235, 895-907.

Roeth, J. F., Sawyer, J. K., Wilner, D. A. and Peifer, M. (2009). Rab11 helps maintain apical crumbs and adherens junctions in the Drosophila embryonic ectoderm. PLOS ONE 4, e7634.

Roh, M. H., Makarova, O., Liu, C. J., Shin, K., Lee, S., Laurinec, S., Goyal, M. Wiggins, R. and Margolis, B. (2002). The Maguk protein, Pals1, functions as an adapter, linking mammalian homologues of Crumbs and Discs Lost. J. Cell Biol. 157, 161-172.

Shin, K., Straight, S. and Margolis, B. (2005). PATJ regulates tight junction formation and polarity in mammalian epithelial cells. J. Cell Biol. 168, 705711.

St Johnston, D. and Ahringer, J. (2010). Cell polarity in eggs and epithelia: parallels and diversity. Cell 141, 757-774.

Sugihara-Mizuno, Y., Adachi, M., Kobayashi, Y., Hamazaki, Y., Nishimura M., Imai, T., Furuse, M. and Tsukita, S. (2007). Molecular characterization of angiomotin/JEAP family proteins: interaction with MUPP1/Patj and their endogenous properties. Genes Cells 12, 473-486.
Tanentzapf, G., Smith, C., McGlade, J. and Tepass, U. (2000). Apical, lateral, and basal polarization cues contribute to the development of the follicular epithelium during Drosophila oogenesis. J. Cell Biol. 151, 891-904

Tepass, U., Theres, C. and Knust, E. (1990). crumbs encodes an EGF-like protein expressed on apical membranes of Drosophila epithelial cells and required for organization of epithelia. Cell 61, 787-799.

Wells, C. D., Fawcett, J. P., Traweger, A., Yamanaka, Y., Goudreault, M. Elder, K., Kulkarni, S., Gish, G., Virag, C., Lim, C. et al. (2006). A Rich1/Amot complex regulates the $\mathrm{Cdc42}$ GTPase and apical-polarity proteins in epithelial cells. Cell 125, 535-548.

Wodarz, A., Hinz, U., Engelbert, M. and Knust, E. (1995). Expression of crumbs confers apical character on plasma membrane domains of ectodermal epithelia of Drosophila. Cel/ 82, 67-76.

Wodarz, A., Ramrath, A., Kuchinke, U. and Knust, E. (1999). Bazooka provides an apical cue for Inscuteable localization in Drosophila neuroblasts. Nature $\mathbf{4 0 2}$ 544-547.

Xu, N., Keung, B. and Myat, M. M. (2008). Rho GTPase controls invagination and cohesive migration of the Drosophila salivary gland through Crumbs and Rho-kinase. Dev. Biol. 321, 88-100.

Zhou, B., Wu, Y. and Lin, X. (2011). Retromer regulates apical-basal polarity through recycling Crumbs. Dev. Biol. 360, 87-95.

Zhou, W. and Hong, Y. (2012). Drosophila Patj plays a supporting role in apicalbasal polarity but is essential for viability. Development 139, 2891-2896. 